\title{
A Review on Speckle Noise Reduction Techniques
}

\author{
Er. Kritika Bansal ${ }^{1}$, Er. Akwinder kaur ${ }^{2}$ \\ Department of Computer Science Engineering, Chandigarh University \\ Research Scholar ${ }^{l}$, Assistant Professor ${ }^{2}$
}

\begin{abstract}
In medical image processing, Image denoising plays a very important role in diagnosis. Medical images are very much inconsistent. In medical imaging, Ultrasound is very popular due to its low cost, least harmful to human body, real time system and small in size. Besides itsadvantage,It suffers from a major problem i.e. speckle noise which reduces the diagnosis ability. Speckle noise is the result of diffuse scattering, which occurs when the sound waves (ultrasound) randomly interferes with a small particle or object on a scale comparable to small wavelength. Speckle Noise is an inherent property of SAR and ultrasound images. As Speckle noise is multiplicative in nature which is very much difficult to remove.So this paper presents a review on different spatial and transform methods such as spatial domain technique and wavelet domain techniques which are already proposed for filtering speckle noise in ultrasound images

Keywords: Speckle Noise, Ultrasound images, Multiplicative noise, denoising, filters
\end{abstract}

\section{Introduction}

Each imaging system suffers with a common problem of "noise" unwanted data which may reduce the contrast, shape size of object in an image and blurring of edges in an image is terms as "noise". Medical images are corrupted by noise due to its acquisition and transmission. There are basically two types of models Additive and Multiplicative. Additive can be easily handled and it is easy to remove. Whereas multiplicative noise is image dependent, complex and difficult to remove. When multiplicative noise is caused by de-phased echoes from which it appears is terms as "speckle noise". Speckle noise may appear distinct different in different imaging system. But it is always in a granular pattern due to the formation of coherent waves.So the different speckle filters are there to improve the image. Different methods are used to eliminate speckle noise, basicallyall mathematical models it depends on three fundamental assumptions (1) Speckle noise in SAR is a multiplicative noise. (2) The signal and the noise are statistically independent of each other. (3) The sample mean and variance of a single pixel are equal to the mean and variance of the local area that is centered on that pixel. So there are two different ways of designing the filter one is spatial domain and other is transform domain.

\section{Statistical Model of Speckle in Ultrasound}

Much literature has been published on the speckle noise in ultrasound among them the multiplicative model is proved to most accurate and suitable for Ultrasound imaging. A speckle image can be expressed as

$$
g=f^{*} n \text {. }
$$

Where fis the original image and the noise $n$ is assumed to have a unitary mean and an unknown variance

\section{Speckle Filtering Methods}

There are basically two speckle filtering methods. Both these methods have their own advantages and disadvantages and are adopted according to their specific requirements complexity and performance.

1. Spatial domain technique

2. Wavelet domain techniques

\subsection{Spatial domain filtering}

Spatial domain techniques are widely used in the digital image processing. The term spatial refers to the grid of pixels that represents an image. The relative position and local neighbourhood pixel values are important in spatial domain techniques. So this paper describes some filters for speckle noise reduction.

\subsubsection{Box Filter:}

A spatial averaging filter in which all coefficients are equal is called a box filter. These types of filters are used for blurring and for noise reduction. The output of such a linear smoothing filter is simply the average of the pixels in the neighbourhood of the pixel mask. By replacing the value of every pixel in an image by the 
average of the gray levels in the neighbourhood of the filter mask, the process results in an image with reduced sharp transitions in gray levels.

Advantage: this type of filter is used in noise reduction and smoothing.

Disadvantage: this type of filter can blur the edges

\subsubsection{Median Filter}

Median filter is a non linear filtering technique for speckle noise reduction. It works best with salt and pepper noise while retaining sharp edges in an image. This filter sorts the surrounding pixel values in the window to an orderly set and replaces the centre pixel value with the middle value.

Advantages:

1 .Median filter is more robust than mean filterso a single very unrepresentative pixel in a neighbourhood will not affect the median value significantly

2. It is better edge preserving filter

The major disadvantage is the extra computation time is needed to sort the intensity value of all set.

\subsubsection{Statistic Lee Filter}

This lee based is based on the approach. It depends upon the variance. If variance over an area is low or constant then the smoothing will occurs. If the variance is high then the smoothing will not be performed. The lee filter assumes that speckle noise is multiplicative. Than the SAR image can be approximated by a linear model gives the eqn

$\operatorname{Img}(\mathrm{i}, \mathrm{j})=I_{m}+\mathrm{W} *\left(C P-I_{m}\right)$

The major disadvantage of Lee filter is that it ignores the speckle noise in the areas closest to edges and lines

\subsubsection{Statistic Kuan Filter}

The Kuan filter is better than Lee filter .it does not make an approximation on the noise variance within filter window. It just simplifies to convert the multiplicative noise model in to additive linear form. But it depends on the ENL from an image to determine a weighting function $\mathrm{W}$ gives in an eqn to perform filtering. $\mathrm{W}=(1-\mathrm{Cu} / \mathrm{Ci}) /(1+\mathrm{Cu})$

The weighting function is computed from noise variation coefficient of an image, $\mathrm{Cu}$ gives in eqn

$C u=\sqrt{1 / E N L}$

Where ENL is the Equivalent No of Looks

The limitation of Kuan filter is that ENL is needed for computation.

\subsubsection{Frost Filter}

The Frost filter is an Adaptive filter that incorporates the local image statistics in the filtering process assuming a negative distribution for noise. A frost filter adapts to noise variance within the window by applying exponentially weighting factors $\mathrm{M}$ as given in an eqn these weighted functions decrease the variance and window filter reduces. The frost filter has no user defined parameters.

The major limitation of frost filter is that the parameters are adjusted according to variance in each area. If variance is low then the smoothingwill occurs. If variance is high than little smoothing occurs and edges are retained.

\subsubsection{Srad Filter}

It is a Non linear anisotropic diffusion technique, speckle reducing anisotropic diffusion, for removing multiplicative noise in image. This method relies on the instantaneous coefficient of variation (ICOV) edge detector as a controller of diffusion rate near edges of regional structures.

\subsection{Transform Domain Techniques}

In transform domain techniques firstly the image is transformed into frequency domain than it will transform in to spatial domain and then domain specific properties are exploited to process the image. 


\subsubsection{Fourier Transform}

The Fourier transform is the important tool in the image processing. In Fourier transform the image is transformed into sine and cosine function the output of this is in the form of Fourier or frequency transform while the input is in spatial domain. In Fourier transform each point contains particular frequency components which are contained in spatial domain. Now after transforming the image into frequency domain then the frequency components are manipulated according to their noise characterstics.than after manipulation it will transformed into spatial domain by taking the inverse of Fourier transform. In Fourier transform it can only deal with frequency components that is it can deal with time

\subsubsection{Wavelet Transform}

Wavelet provides a Multiresolutional, Sparse Representation, Localization, and EdgeClustering .the use of wavelet is used in last decade. Wavelets provides compression and reduction techniques. It is independent on both space and time. By decomposed the corrupted images into the respective sub-bands by applying the discrete wavelet transform on the input noisy image. Then compute the Thresholding and then applying the idwt to reconstruct the original image. Wavelet is just like a "wave".A wave is an oscillating function of time or space andit isalways periodic.Wavelets are localized waves. In Wavelet firstly the signal to be analysed than multiplied with a wavelet function and then the transform is computed for each and then segment is generated. The Wavelet at high frequencies,gives good time resolution and at low frequenciesthe wavelet transform provides poor resolution.

\subsubsection{Wavelet domain noise filtering}

Since the discrete wavelet transform (DWT) corresponds to basis decomposition, it provides a unique representation of the signal. So in two levels decomposition in wavelet an image is divided in to No of four blocks such as LL,LH,HL, and HH. The LL band is known as approximation band and HH, HL, LH is known as detailed coefficients. They add the high-frequency detail to the approximation image. The wavelet transform first performs one step of the transform on all rows. This Process contains a matrix where the left side contains down sampled low passcoefficients of each row, and the right side contains the highpass coefficients. Then next step of decomposition is applied to all columns. So LL filter is a low pass filter in Image decomposition by using DWT Columns.[1]

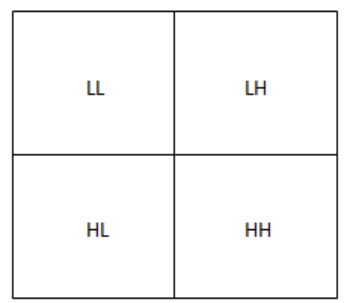

Fig.1. Sub bands of one level 2D wavelet transform

\begin{tabular}{|c|c|c|}
\hline$L L_{n}$ & $H L_{n}$ & \multirow{2}{*}{$H L_{n-2}$} \\
\cline { 1 - 2 }$L H_{n}$ & $H H_{n}$ & \\
\hline & & \\
& & \\
& & \\
& & \\
& & \\
\hline
\end{tabular}

Fig.2.Sub bands of $\mathrm{n}$ levels 2D wavelet transform

So it provides rough description on in an image. Whereas HL, LH contains the one side high pass filtered in one direction and other side contains low pass in another direction. And $\mathrm{HH}$ is the high pass filtered in both directions. So in this way it contains high frequency components.

\subsection{Thresholding}

Thresholding is for image Segmentation. Thresholding is used to remove the noise in an image or to improve the quality of images. It is used to preserve the edges in images. There are basically two types of Thresholding techniques 
i. Global Thresholding algorithms

ii. Local and adaptive Thresholding algorithms

In global Thresholding a single value for entire pixel is used. In local or adaptive Thresholding different threshold values for different local areas are used.

Thresholding is of two types

i. Hard Thresholding

ii. Soft Thresholding

Hard Thresholding: Hard Thresholding is applied to the detailed coefficients (horizontal, vertical and diagonal)after applying the dwt of the image. In hard Thresholding the values should be compared with given threshold value. If the hard Thresholdingvalue is lower than that threshold value than it sets to be zero. Hard Thresholding is "keep or kill".In hardThresholding exhibits the unwanted accessional fluctuation. So Soft Thresholding is used.

Soft Thresholding: It involves first setting to zero the elements whose absolute values are lower than the threshold and then scaling the non-zero coefficients towards zero. Soft Thresholdingeliminates the discontinuity that is inherent in hard Thresholding. In soft Thresholding there is a range of wavelet coefficients.. Soft Thresholdingsmoothes the images as well as preserve the images as compared to hard Thresholding.

\section{Conclusion:}

As discussed in this paper, different filtering methods have been used traditionally to lessen the speckle noise. But, due to some disadvantages in all the discussed techniques, wavelet transform techniques are now used to counter all their disadvantages. As wavelet transform is independent of fixed window size as it is frame based approach, it gives us a better resolution images and hence provides better results.

\section{References}

[1] Adib Akl, Charles Yaacoub "A hybrid filters for image despeckling with wavelet-based denoising and spatial filtering"IEEE International Conference on Communication Systems and Network Technologies, 2013.

[2] O.V. Michailovich, A. Tannenbaum, "Despeckling of medical ultrasound images", IEEE Transactions on Ultrasonic's, Ferroelectrics and Frequency Control, pp. 64-78, Jan. 2006.

[3] D.H.H. Santosh, V. N. L. Kumar, D. R. Ramesh, P. S. Prasad, "Efficient Techniques for Denoising of Speckle and Highly Corrupted Impulse Noise Images", IEEE Transactions on Electronics and Computer Technology (ICECT), Vol.3, pp 253-257, 2011.

[4] R. Ahirwar and A. Choubey, "A Novel Wavelet-based Denoising Method of SAR Image Using Interscale Dependency", 2011 International Conference on Computational Intelligence and Communication Systems, Gwalior, India, Oct. 2011.

[5] Inderjeet Singh, Er.Lal Chand "Speckle Noise Reduction based on Discrete Wavelet Transform" International Journal of Computer Trends and Technology IJCTT 2013.

[6] S.Sudha, G.R.Suresh and R.Sukanesh "Speckle Noise Reduction in Ultrasound Images by Wavelet Thresholding based on Weighted Variance" International Journal of Computer Theory and Engineering, 2009.

[7] NidhiChandrakar, Devanand Bhonsle "A New Hybrid Image Denoising Method using Bilateral Filter and DWT"International Journal of IT, Engineering and Applied Sciences Research 2013.

[8] K.Karthikeyan, Dr. C. Chandrasekhar "Speckle Noise Reduction of Medical Ultrasound Images using Bayes shrink Wavelet Threshold" International Journal of Computer Applications 2011.

[9] R. Eveline Pregitha, Dr. V. Jegathesan "Speckle noise reduction in ultrasound fetal images using an edge preserving adaptive shock filter" International Journal of Scientific and Research Publications 2012.

[10] Udomhunskal, Wongsita "Ultrasonic speckle denoising using the combination of wavelet transform and Wiener filter" IEEE International Conference on Computational Intelligence and Computing Research 2010

[11] Sabahaldin A. Husain, Sami M. Ghorshi "Image Denoising based on Spatial Wavelet Filter using Hybrid Thresholding Function" International Journal of Computer Applications 2012 